\title{
A Method of Auxiliary Sources Approach for Modelling the Impact of Ground Planes on Antenna
}

Larsen, Niels Vesterdal; Breinbjerg, Olav

Published in:

Proceedings of the European Conference on Antennas and Propagation

Link to article, DOI:

10.1109/EUCAP.2006.4584526

Publication date:

2006

Link back to DTU Orbit

Citation $(A P A)$ :

Larsen, N. V., \& Breinbjerg, O. (2006). A Method of Auxiliary Sources Approach for Modelling the Impact of Ground Planes on Antenna. In Proceedings of the European Conference on Antennas and Propagation (pp. 14). IEEE. https://doi.org/10.1109/EUCAP.2006.4584526

\section{General rights}

Copyright and moral rights for the publications made accessible in the public portal are retained by the authors and/or other copyright owners and it is a condition of accessing publications that users recognise and abide by the legal requirements associated with these rights.

- Users may download and print one copy of any publication from the public portal for the purpose of private study or research.

- You may not further distribute the material or use it for any profit-making activity or commercial gain

- You may freely distribute the URL identifying the publication in the public portal 


\title{
A METHOD OF AUXILIARY SOURCES APPROACH FOR MODELLING THE IMPACT OF GROUND PLANES ON ANTENNA RADIATION
}

\author{
Niels Vesterdal Larsen* and Olav Breinbjerg \\ Ørsted•DTU, Technical University of Denmark, Ørsteds Plads build. 348, DK-2800 Kgs. Lyngby, Denmark. \\ nvl@oersted.dtu.dk,ob@oersted.dtu.dk
}

\begin{abstract}
A Method of Auxiliary Sources (MAS) approach is proposed to model the impact of finite ground planes on the radiation from antennas. Taking outset in a priori known antenna characteristics a scattering problem is formed in which the antenna is modelled by an incident field and the ground plane as a scatterer. Two different antenna test cases are shown and the calculated results agree well with reference measurements.
\end{abstract}

Key words: Antenna radiation, finite ground planes, computational electromagnetics.

\section{INTRODUCTION}

When employing commercially available software in antenna design the computational complexity often prohibits the inclusion of the antenna surroundings such as a large finite ground plane. In many cases antenna designs are thus based on the assumptions of an infinite ground plane, or no ground plane, and hence the impact of a finite ground plane on the antenna radiation is not always known prior to actual fabrication and testing of the antenna. However, from such analyses certain antenna characteristics, e.g., impedance, currents on wires, or fields in apertures, can typically be extracted. From these characteristics the antenna can be approximately represented by an impressed incident field whose interaction with the ground plane forms a scattering problem. In principle this requires that the antenna characteristics can be assumed to be unaffected by the introduction of the finite ground plane which, however, is a reasonable assumption for large ground planes. In the present work the Method of Auxiliary Sources (MAS) is employed to assess the influence of such finite ground planes. Indeed for many large-scale problems the potential of MAS has been well documented, e.g., as shown in [1]. In this text examples of the approach are given in virtue of two practical antenna cases. These are investigated with ground planes of different shapes and sizes and the obtained far-field results are compared to reference measurements.

\section{THE MAS MODEL}

The mathematical formulation of the MAS model takes outset in a scattering problem where an impedance scatterer, i.e., the finite ground plane, with the surface impedance $Z_{s}$ is surrounded by free space with intrinsic impedance $Z_{0}$. The outward pointing unit normal vector of the ground plane surface $B$ is denoted by $\hat{\mathbf{n}}$. The ground plane is illuminated by a time-harmonic incident field $\left(\mathbf{E}^{i}, \mathbf{H}^{i}\right)$, with angular frequency $\omega$ and freespace wave number $k_{0}=\frac{2 \pi}{\lambda}$, where $\lambda$ is the wavelength. The time dependence $e^{j \omega t}$ is assumed and suppressed throughout the text. The standard impedance boundary condition (SIBC) [2] is assumed to hold on $B$ and thus the total field $\left(\mathbf{E}=\mathbf{E}^{s}+\mathbf{E}^{i}, \mathbf{H}=\mathbf{H}^{s}+\mathbf{H}^{i}\right)$ being the sum of the incident field and the scattered field $\left(\mathbf{E}^{s}, \mathbf{H}^{s}\right)$ satisfies the relation

$$
\hat{\mathbf{n}} \times \mathbf{E}=Z_{s} \hat{\mathbf{n}} \times(\hat{\mathbf{n}} \times \mathbf{H}) .
$$

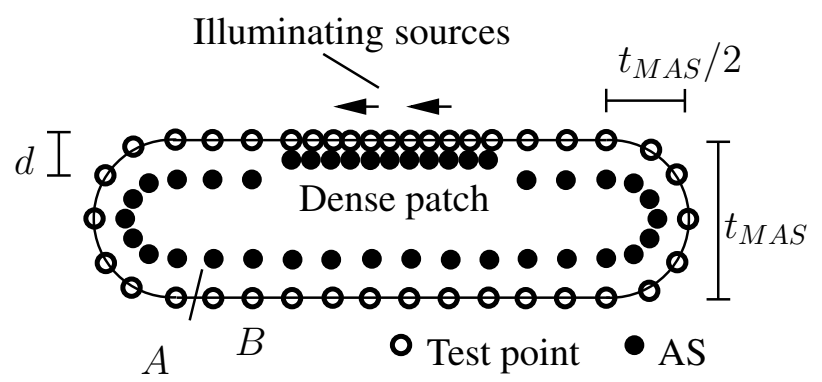

Figure 1. Cross-sectional view of a MAS ground plane with rounded edges and a dense patch of $A S$.

In order to recover the scattered field $N$ auxiliary sources (AS) are positioned on an auxiliary surface $A$ inside the ground plane as shown in Figure 1. The AS are chosen as pairs of crossed Hertzian dipoles of either electric or magnetic type with independent excitations. The SIBC (1) is tested in $M=N$ test points (TP) on $B$. $A$ is chosen conformal to $B$ and receded into the ground plane by a distance $d$. The scattered field at the $m$ 'th TP, is the sum 
of the fields from the AS

$$
\left\{\begin{array}{c}
\mathbf{E}_{m}^{s} \\
\mathbf{H}_{m}^{s}
\end{array}\right\}=\sum_{n=1}^{N}\left[C_{n}^{\langle 1\rangle}\left\{\begin{array}{c}
\mathbf{E}_{n m}^{\langle 1\rangle} \\
\mathbf{H}_{n m}^{\langle 1\rangle}
\end{array}\right\}+C_{n}^{\langle 2\rangle}\left\{\begin{array}{c}
\mathbf{E}_{n m}^{\langle 2\rangle} \\
\mathbf{H}_{n m}^{\langle 2\rangle}
\end{array}\right\}\right]
$$

where $\left(\mathbf{E}_{n m}^{\langle\cdot\rangle}, \mathbf{H}_{n m}^{\langle\cdot\rangle}\right)$ is the field from the $n$ 'th AS evaluated at the $m$ 'th TP and superscripts $\langle 1\rangle,\langle 2\rangle$ refer to the two crossed Hertzian dipoles constituting the AS. Insertion of (2) in (1) yields $2 M$ equations with $2 N$ unknowns for the two components of the boundary condition to be satisfied, and from this the MAS excitation coefficients $C_{n}^{\langle 1\rangle}$ and $C_{n}^{\langle 2\rangle}$ are determined.

It is well known that the standard MAS formulation [3], which is used here, does not lend itself well to modelling of thin structures or structures with sharp edges. Moreover, in cases where the illuminating sources are very close to the ground plane the variation of the incident field along the surface is particularly high and may cause inaccuracy. Both of these potential problems can in principle be solved by employing a localised Method of Moments solution at the corners and near closely positioned sources such as reported in [4, 5]. This, however, will be at the expense of increased complexity. The so-called Modified MAS [6] is another alternative which has been shown to be effective for thin structures. By using rounded edges in the MAS ground plane model, the simple standard MAS can also be used to model ground planes. Although this introduces a slight difference as compared to the true ground plane the impact of this is not very large as will be shown in the examples to follow.

In this work the ground plane models are implemented with rounded edges and the thickness of the ground plane models, denoted $t_{M A S}$, is therefore larger than for the ground planes themselves. Also the edges become semicircular with a curvature radius of $t_{M A S} / 2$. The size of the ground plane model is defined with respect to the flat part and thus the rounded edges constitute small additions to the overall ground plane size. In the case of closely positioned illuminating sources a patch of densely positioned AS is introduced in the vicinity below the illuminating sources and furthermore the distance between $B$ and $A$ is $d / 2$ in this area. A sketch of a MAS ground plane model with rounded edges and with a dense patch of AS is shown in Figure 1. Due to the aforementioned limitation in the standard MAS, it is clear that there is a lower limit on the value of $t_{M A S}$ that can be used in the model.

\section{APPLICATIONS AND DISCUSSION}

The MAS formulation is applied to two different antenna configurations where a total of three different ground planes are investigated. Corresponding reference measurements have been conducted for all cases at the DTUESA Spherical Near-Field Antenna Test Facility at the Technical University of Denmark [8]. In the cases shown

\begin{tabular}{|c|c|c|c|}
\hline & GP 1 & GP 2 & GP 3 \\
\hline Shape & Square & Circular & Rounded circ. \\
\hline Dim. & $1.32 \lambda(\mathrm{SL})$ & $7.90 \lambda(\mathrm{D})$ & $5.27 \lambda(\mathrm{D})$ \\
\hline$t_{M A S}$ & $0.25 \lambda$ & $0.5 \lambda$ & $0.5 \lambda$ \\
\hline$N$ & $\sim 750$ & $\sim 4800$ & $\sim 3000$ \\
\hline
\end{tabular}

Table 1. Description of the 3 different ground planes used for the 2 antenna configurations. The dimensions are indicated by side lengths $(S L)$ and diameter $(D)$.

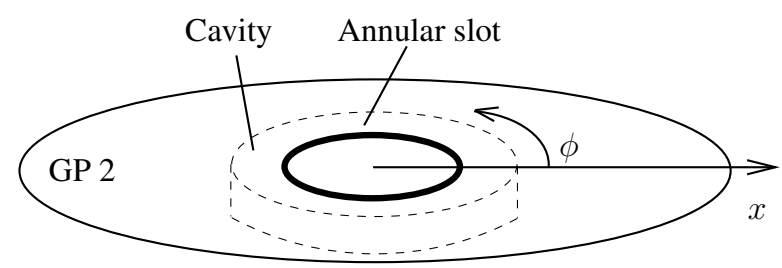

Figure 2. Top/side-view sketch of the cavity-backed annular slot antenna with GP 2.

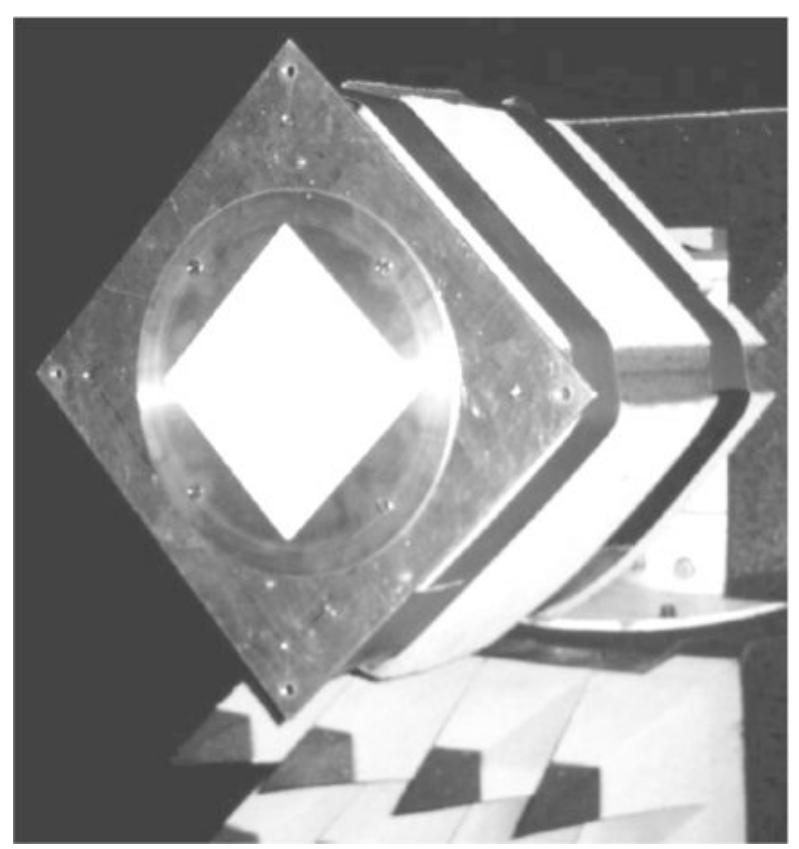

Figure 3. The cavity-backed annular slot antenna mounted with GP 1. The white dielectric superstrate covers the slot.

here the ground planes are all modelled as perfect electric conductors, i.e., $Z_{s}=0$, however, the MAS model of course allows for more general impedance ground planes.

The first antenna case, a cavity-backed annular slot antenna, is described in detail in [7]. Presently it suffices to mention that it is a circularly polarised dual feed antenna fed in phase quadrature at two points spaced $90^{\circ}$ apart along the slot. Based on the measured 2-port scat- 

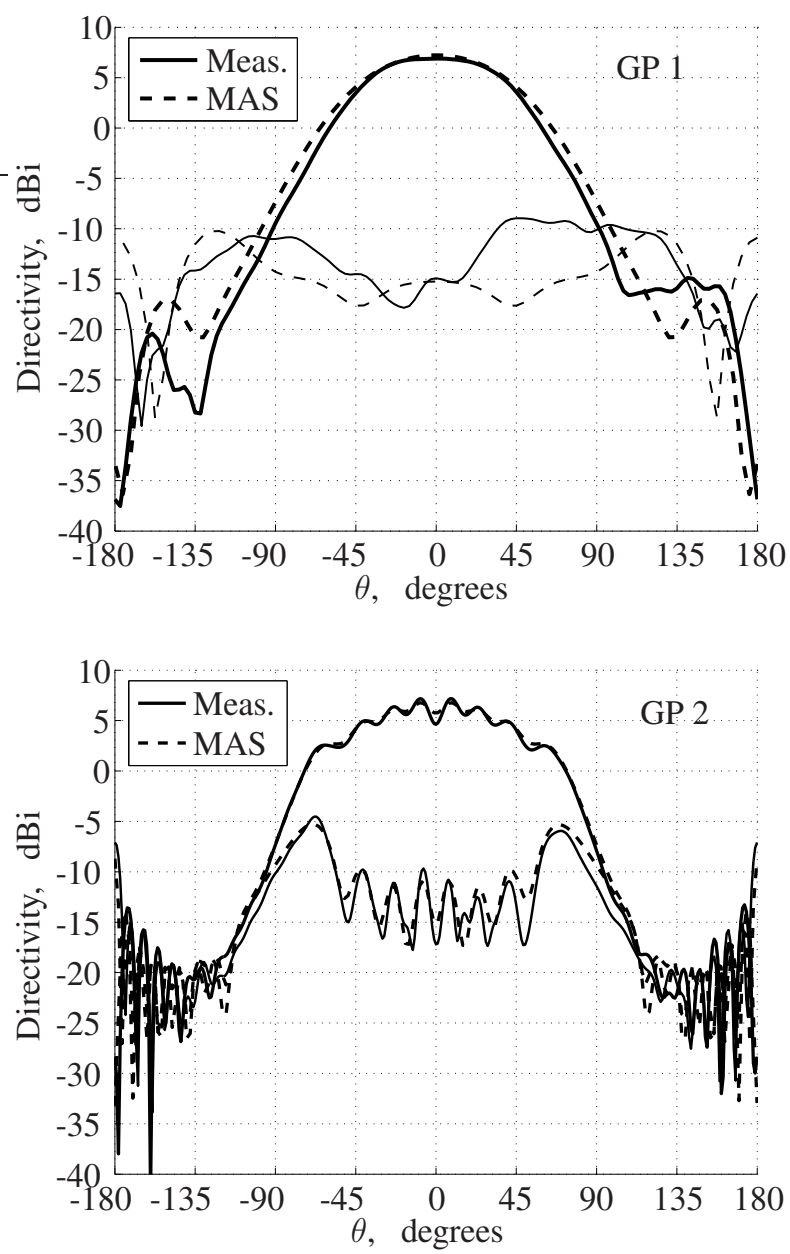

Figure 4. Directivity of the slot antenna mounted on GP 1 (top) and GP 2 (bottom). For both cases $f=1.58 \mathrm{GHz}$ and $\phi=45^{\circ}$.

tering matrix of this antenna the terminal voltages at the two feed points have been determined. Approximation of the slot by a magnetic ring current allows a reasonably accurate representation of the slot and hence the incident field.

Two different ground planes, denoted GP 1-2, are used with this antenna, the details of which are summarised in Table 1. GP 1 is square with side lengths of $1.32 \lambda$, and GP 2 is circular with a diameter of $7.90 \lambda$. The configuration is sketched in Figure 2 and in Figure 3 the cavitybacked annular slot antenna is shown mounted on GP 1 . Both ground planes have sharp edges and a thickness of $0.01 \lambda$. With respect to the MAS model, the thicknesses $t_{M A S}$ and the numbers $N$ of AS employed are given in Table 1. The measurement and simulation results are shown in Figure 4 for the frequency $f=1.58 \mathrm{GHz}$. Generally there is good agreement between the measured and simulated result both with respect to the co- and crosspolar components, particularly for the large GP 2. Some minor deviations are, however, seen between the respec-

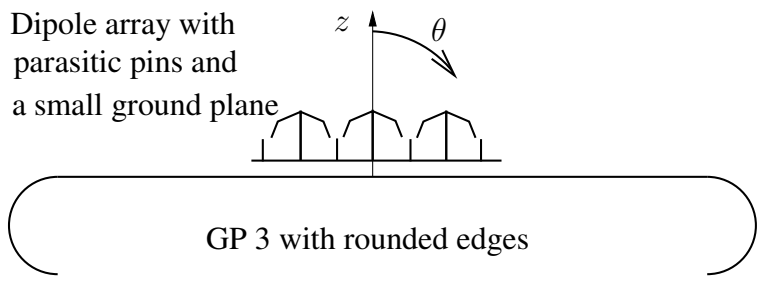

Figure 5. Side-view sketch of the 7-element printed dipole phased array raised above GP 3 .

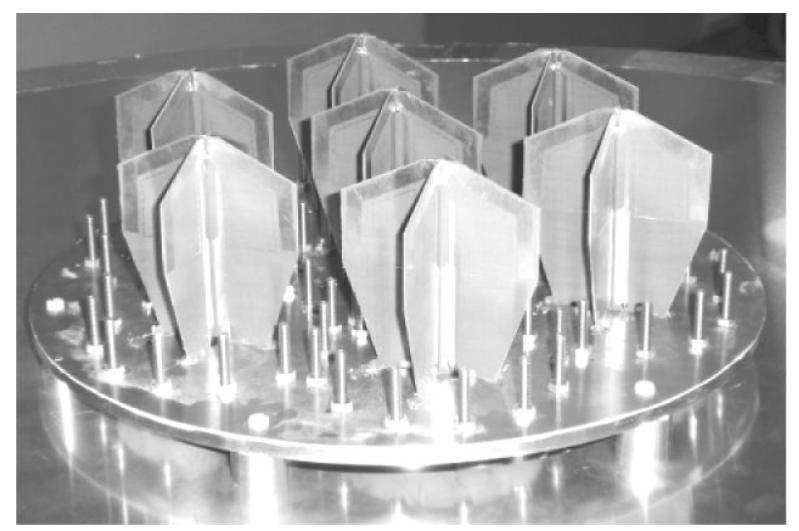

Figure 6. The 7-element printed dipole phased array raised above GP 3 .

tive levels of ripples in the patterns of GP 2. These deviations are partly caused by the approximate model of the slot as well as the rounding of the ground plane edges.

The second case consists of a small phased array antenna comprising 7 circularly polarised crossed printed dipole elements, parasitic pins positioned between the elements, and a small ground plane. The elements are fed at four ports each, resulting in a total of 28 ports in the array. The array with its small ground plane is mounted above a larger circular ground plane GP 3, see Table 1, and raised $0.11 \lambda$ above this. The array configuration is sketched in Figure 5 and in the real array is shown in Figure 6. The diameter of GP 3 is $5.27 \lambda$. The array was initially modelled with the programme AWAS [9] using circular wires for the dipoles as well as a wire grid structure representing the small ground plane; all this raised above an infinite ground plane. From the calculated wire currents the entire array was represented using approximately 5000 electric Hertzian dipoles thus forming the incident field.

Contrary to GP 1-2, GP 3 has rounded edges enabling a more accurate representation in the MAS model. Radiation patterns are shown in Figure 7 for three different scan angles. Both simulations and measurements are shown. The agreement is reasonably good and it is noted that the impact of the finite ground plane on the shape of the main lobe is well recovered. However, it is also noted that some deviations are present particularly for the crosspolar component. The deviations are most likely caused by the differences between the actual array and the wire model from which the incident field is derived. Particu- 

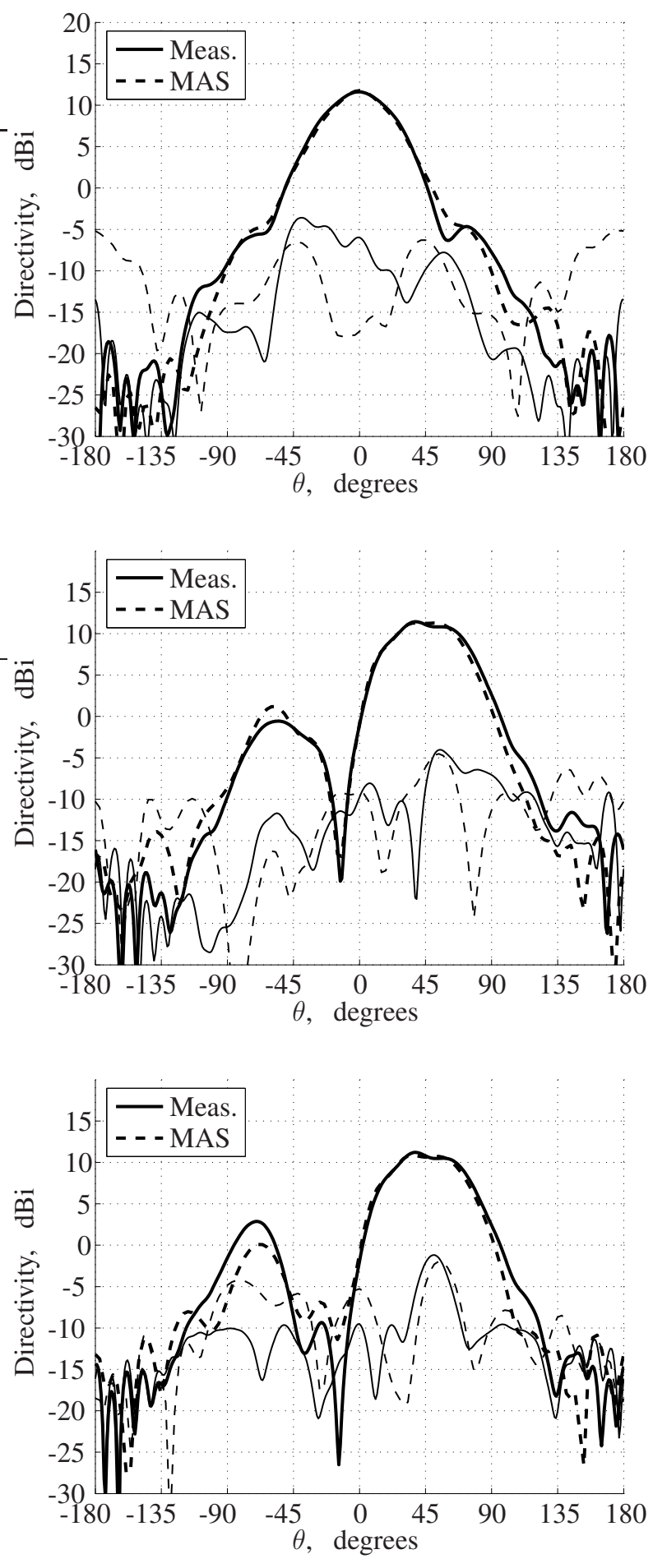

Figure 7. Directivity of the array mounted on GP 3 for $f=1.6 \mathrm{GHz}$. The beam is scanned towards $\theta=0^{\circ}$ (top), $\theta=45^{\circ}, \phi=0^{\circ}$ (middle), and $\theta=45^{\circ}, \phi=90^{\circ}$ (bottom).

larly the differences between the actual printed dipoles, see Figure 6, and the simulated wires may cause this, however, also the wire grid model of the small ground plane may introduce inaccuracy.

\section{CONCLUSION}

The Method of Auxiliary Sources has been applied to the problem of modelling the impact of finite ground planes on the radiation from antennas. Two different antenna cases, a cavity-backed annular slot antenna and a 7-element crossed printed dipole phased array, have been investigated. Both square and circular ground planes with widths of up to $7.90 \lambda$ have been simulated. The results were compared to measurements and good agreement was found. The method can thus be used favourably in combination with commercially available antenna simulation tools, whose computational cost does not allow inclusion of a large finite ground plane.

\section{REFERENCES}

[1] Zaridze R.S., et al., "Electromagnetic Analysis for Vehicle Antenna Development Using Method of Auxiliary Sources", IEEE AP-S Int. Symp., 2004, Vol. 1, pp. 185-188.

[2] Senior T.B.A. and Volakis J.L., "Approximate Boundary Conditions in Electromagnetics", The Institution of Electrical Engineers, London 1995.

[3] Kaklamani D.I. and Anastassiu H.T., "Aspects of the Method of Auxiliary Sources in Computational Electromagnetics", IEEE Antennas and Propagat. Mag., Vol. 44, No. 3, 2002, pp. 48-64.

[4] Eisler S. and Leviatan Y., "Analysis of Electromagnetic Scattering from Metallic and Penetrable Cylinders with Edges Using a Multifilament Current Model", IEE Proc., Vol. 136, Pt. H, No. 6, 1989, pp. 431-438.

[5] Larsen N.V. and Breinbjerg O., "A Hybrid MAS/MoM Technique for 2D Impedance Scatterers Illuminated by Closely Positioned Sources", Microwave Opt. Technol. Lett., Vol. 44, No. 2, 2005, pp. 112-114.

[6] Shubitidze F., Anastassiu H.T., and Kaklamani D.I., "An Improved Accuracy Version of the Method of Auxiliary Sources for Computational Electromagnetics", IEEE Antennas and Propagat. Mag., Vol. 52, No. 1, 2004, pp. 302-309.

[7] Larsen N.V. and Breinbjerg O., "An L-band, Circularly Polarised, Dual-Feed, Cavity-Backed Annular Slot Antenna for Phased Array Applications", Microwave Opt. Technol. Lett., Vol. 48, No. 5, 2006, pp. 873-878.

[8] Homepage: http://www.oersted.dtu.dk/English/research/emi/ afg/dtu_esa_facility.aspx

[9] Djordjević A.R., et al., AWAS for Windows, Version 2.0, Analysis of Wire Antennas and Scatterers, Software and User Manual, Artech House, Inc., Boston, 2002. 